\title{
On Initial-Boundary Value Problems for Hyperbolic Equations in Domains with Conical Points
}

\author{
Nguyen Manh Hung and Nguyen Thanh Anh \\ Department of Mathematics, Hanoi National University of Education, No. 136 Xuan Thuy Street, Hanoi, Vietnam \\ Correspondence should be addressed to Nguyen Thanh Anh; thanhanh@hnue.edu.vn
}

Received 24 December 2012; Accepted 14 July 2013

Academic Editor: Ağacik Zafer

Copyright (C) 2013 N. M. Hung and N. T. Anh. This is an open access article distributed under the Creative Commons Attribution License, which permits unrestricted use, distribution, and reproduction in any medium, provided the original work is properly cited.

We consider an initial-boundary value problem for general higher-order hyperbolic equation in an infinite cylinder with the base containing conical points on the boundary. We establish several results on the unique solvability, the regularity, and the asymptotic behaviour of the solution near the conical points.

\section{Introduction}

A large number of investigations have been devoted to boundary value problems in nonsmooth domains with conical points. Up to now, elliptic boundary value problems in domains with point singularities have been thoroughly investigated (see, e.g., [1-3]).

We are concerned with hyperbolic equations in domains with conical points. This topic has been investigated in many works with different approaches. For example, [4, 5] the Cauchy-Dirichlet and Cauchy-Neumann problems for second-order hyperbolic systems with the coefficients independent of the time variable were treated in which the asymptotics of the solutions were established with explicit formulas for the coefficients. In [6-9], initial-boundary value problems for general higher-order hyperbolic equations and systems with the coefficients depending on both spatial and time variable in a domain containing conical points were studied in which the unique solvability, the regularity, and the asymptotic behaviour of the solutions near the conical points were obtained. In the present paper, these results are extended to initial-boundary value problems for general higher-order hyperbolic equations with more general boundary conditions in infinite cylinders with the bases containing conical points. Such boundary conditions have been considered for elliptic equations in $[10,11]$ and for parabolic equations in $[12,13]$.

Our paper is organized as follows. Section 2 is devoted to some notations and the formulation of the problem. The main results will be stated in Section 3. The proofs of the main theorems will be given in Sections 4 and 5 .

\section{Notations and the Formulation of the Problem}

Let $G$ be a bounded domain in $\mathbb{R}^{n}(n \geqslant 2)$ with the boundary $\partial G$. We suppose that $\Gamma=\partial G \backslash\{0\}$ is a smooth manifold and $G$ in a neighborhood $U$ of the origin 0 coincides with the cone $K=\{x: x /|x| \in \Omega\}$, where $\Omega$ is a smooth domain on the unit sphere $S^{n-1}$ in $\mathbb{R}^{n}$. For each $T, 0<T \leqslant \infty$, denote $Q_{T}=$ $G \times(0, T), G_{T}=G \times\{T\}$. Specifically, we set $Q=Q_{\infty}=G \times \mathbb{R}_{+}^{*}$, $S=\Gamma \times \mathbb{R}_{+}$, where $\mathbb{R}_{+}^{*}=(0, \infty), \mathbb{R}_{+}=[0, \infty)$. For each multiindex $\alpha=\left(\alpha_{1}, \ldots, \alpha_{n}\right) \in \mathbb{N}^{n}$, set $|\alpha|=\alpha_{1}+\cdots+\alpha_{n}$ and $\partial_{x}^{\alpha}=\partial_{x}^{\alpha}=\partial_{x_{1}}^{\alpha_{1}} \cdots \partial_{x_{n}}^{\alpha_{n}}$. For a nonnegative integer $k$ we write $u_{t^{k}}$ instead of $\partial_{t}^{k} u$.

We introduce the following differential operator:

$$
L u=L(x, t, \partial) u=\sum_{|\alpha|,|\beta| \leqslant m}(-1)^{|\alpha|} \partial^{\alpha}\left(a_{\alpha \beta} \partial^{\beta} u\right),
$$

where $a_{\alpha \beta}=a_{\alpha \beta}(x, t)$ are bounded complex-valued functions defined in $\bar{Q}$. We assume that $a_{\alpha \beta}=(-1)^{|\alpha|+|\beta|} \bar{a}_{\beta \alpha}$ for all $|\alpha|,|\beta| \leqslant m$. This means the differential operator $L$ is formally 
self-adjoint. We assume further that there exists a positive constant $\mu_{0}$ such that

$$
\sum_{|\alpha|=|\beta|=m} a_{\alpha \beta}(x, t) \eta_{\beta} \overline{\eta_{\alpha}} \geq \mu_{0} \sum_{|\alpha|=m}\left|\eta_{\alpha}\right|^{2},
$$

for all $\eta_{\alpha} \in \mathbb{C},|\alpha|=m$ and all $(x, t) \in \bar{Q}$.

We introduce also a system of boundary operators:

$$
B_{j}=B_{j}\left(x, t, \partial_{x}\right)=\sum_{|\alpha| \leqslant \mu_{j}} b_{j, \alpha}(x, t) \partial_{x}^{\alpha}, \quad j=1, \ldots, m,
$$

on $S$ with smooth coefficients in $\bar{Q}$. Suppose that

$$
\begin{gathered}
\operatorname{ord} B_{j}=\mu_{j} \leqslant m-1 \quad \text { for } j=1, \ldots, \chi, \\
m \leqslant \operatorname{ord} B_{j}=\mu_{j} \leqslant 2 m-1 \quad \text { for } j=\chi+1, \ldots, m,
\end{gathered}
$$

and the coefficients of $B_{j}$ are independent of $t$ if $\operatorname{ord} B_{j}<m$, where $\operatorname{ord} B_{j}$ stands for the order of the differential operator $B_{j}$. Suppose that $\left\{B_{j}\left(x, t, \partial_{x}\right)\right\}_{j=1}^{m}$ is a normal system on $S$ for all $t \in \mathbb{R}_{+}$; that is, the two following conditions are satisfied:

(i) $\mu_{j} \neq \mu_{k}$ for $j \neq k$,

(ii) $B_{j}^{\circ}(x, t, v(x)) \neq 0$ for all $(x, t) \in S, j=1, \ldots, m$.

Here $v(x)$ is the unit outer normal to $S$ at point $x$, and $B_{j}^{\circ}\left(x, t, \partial_{x}\right)$ is the principal part of $B_{j}\left(x, t, \partial_{x}\right)$,

$$
B_{j}^{\circ}=B_{j}^{\circ}\left(x, t, \partial_{x}\right)=\sum_{|\alpha|=\mu_{j}} b_{j, \alpha}(x, t) \partial_{x}^{\alpha}, \quad j=1, \ldots, m .
$$

Furthermore, we assume that $B_{j}^{\circ}(0, t, v(x)) \neq 0$ for all $x \in S$ sufficiently close to the origin 0 .

To be able to reduce the problem considered to variational form we assume that there are boundary operators $\Phi_{j}$ on $S$, $j=1, \ldots, m$, such that

$$
\begin{gathered}
B(t, u, v)=\int_{G} L u \bar{v} d x+\sum_{j=1}^{\chi} \int_{S} \Phi_{j} u \overline{B_{j} v} d s \\
+\sum_{j=\chi+1}^{m} \int_{S} B_{j} u \overline{\Phi_{j} v} d s,
\end{gathered}
$$

for all $u, v \in C_{0}^{\infty}(\bar{G} \backslash\{0\})$ and a.e. $t \in \mathbb{R}_{+}$. Here

$$
B(t, u, v)=\sum_{|\alpha|,|\beta|=0}^{m} \int_{G} a_{\alpha \beta}(\cdot, t) \partial_{x}^{\beta} u \overline{\partial_{x}^{\alpha} v} d x, \quad t \in \mathbb{R}_{+},
$$

is the bilinear form associated with the operator $L\left(x, t, \partial_{x}\right)$. Of course this is an essential restriction on the structure of the boundary operators in (3). However, if the system of boundary operators in (3) is a Dirichlet system (then all ord of $B_{j}$ are less than $m$ ) or a generalized Neumann system (then $m \leqslant$ ord $B_{j} \leqslant 2 m-1$ for all $\left.j=1, \ldots, m\right)$, the equality (6) holds for a suitable system $\Phi_{j}, j=1, \ldots, m$ (see $[10$, Section I.7.]).
In this paper, we consider the following problem:

$$
\begin{gathered}
u_{t t}+L u=f \text { in } \mathrm{Q}, \\
B_{j} u=0 \text { on } S, \quad j=1, \ldots, m, \\
\left.u\right|_{t=0}=\left.u_{t}\right|_{t=0}=0 \text { in } G .
\end{gathered}
$$

Before giving the definition of generalized solutions to this problem, let us introduce some needed functional spaces.

Let us denote by $H^{l}(G)(l \in \mathbb{N})$ the usual Sobolev space of all functions $u$ defined in $G$ with the norm

$$
\|u\|_{H^{l}(G)}=\left(\int_{G} \sum_{|\alpha| \leqslant l}\left|D^{\alpha} u\right|^{2} d x\right)^{1 / 2}<\infty .
$$

If $l \geqslant 1$, by $H^{l-(1 / 2)}(\Gamma)$ we denote the space of traces of functions in $H^{l}(G)$ on $\Gamma$ with the norm

$$
\|u\|_{H^{l-(1 / 2)}(\Gamma)}=\inf \left\{\|v\|_{H^{l}(G)}: v \in H^{l}(G),\left.v\right|_{\Gamma}=u\right\} .
$$

We set

$$
H_{B}^{m}(G)=\left\{u \in H^{m}(G): B_{j} u=0 \text { on } S \text { for } j=1, \ldots, \chi\right\},
$$

with the same norm as in $H^{m}(G)$. By $V_{2, \alpha}^{l}(G)(\alpha \in \mathbb{R})$ we denote the closure of $C_{0}^{\infty}(\bar{G} \backslash\{0\})$ with respect to the norm

$$
\|u\|_{V_{2, \alpha}^{l}(G)}=\left(\sum_{|p| \leqslant l} \int_{G} r^{2(\alpha+|p|-l)}\left|D^{p} u\right|^{2} d x\right)^{1 / 2}
$$

where $r=|x|=\left(\sum_{k=1}^{n} x_{k}^{2}\right)^{1 / 2}$. By $H_{\alpha}^{l}(G)$ we denote the weighted Sobolev space of functions $u$ defined in $G$ with the norm

$$
\|u\|_{H_{\alpha}^{l}(G)}=\left(\sum_{|p| \leqslant l} \int_{G} r^{2 \alpha}\left|D^{p} u\right|^{2} d x\right)^{1 / 2}<+\infty .
$$

If $l \geqslant 1$, then $V_{\alpha}^{l-1 / 2}(\Gamma), H_{\alpha}^{l-1 / 2}(\Gamma)$ denote the spaces consisting of traces of functions from respective spaces $V_{2, \alpha}^{l}(G), H_{\alpha}^{l}(G)$ on the boundary $\Gamma$ with the respective norms

$$
\begin{aligned}
& \|u\|_{V_{\alpha}^{l-1 / 2}(\Gamma)}=\inf \left\{\|v\|_{V_{2, \alpha}^{l}(G)}: v \in V_{2, \alpha}^{l}(G),\left.v\right|_{\Gamma}=u\right\}, \\
& \|u\|_{H_{\alpha}^{l-1 / 2}(\Gamma)}=\inf \left\{\|v\|_{H_{\alpha}^{l}(G)}: v \in H_{\alpha}^{l}(G),\left.v\right|_{\Gamma}=u\right\} .
\end{aligned}
$$

Let $X, Y$ be Banach spaces, $0<T \leqslant \infty$. We denote by $L_{2}((0, T), X)$ the space of all functions $u:(0, T) \rightarrow X$ such that

$$
\|u\|_{L_{2}((0, T), X)}=\left(\int_{0}^{T}\|u(t)\|_{X}^{2} d t\right)^{1 / 2}<\infty
$$

and by $H^{1}((0, T), X, Y)$ the space of all functions $u \in$ $L_{2}((0, T), X)$ such that $u_{t} \in L_{2}((0, T), Y)$. The norm in $H^{1}((0, T), X, Y)$ is defined by

$$
\|u\|_{H^{1}((0, T), X, Y)}=\left(\|u\|_{L_{2}((0, T), X)}^{2}+\left\|u_{t}\right\|_{L_{2}((0, T), Y)}^{2}\right)^{1 / 2} .
$$


For some $\gamma \in \mathbb{R}$, we denote by $L_{2}\left(\mathbb{R}_{+}^{*}, X, \gamma\right)$ the space of all functions $u: \mathbb{R}_{+}^{*} \rightarrow X$ such that

$$
\|u\|_{L_{2}\left(\mathbb{R}_{+}^{*}, X, \gamma\right)}=\left(\int_{0}^{\infty}\|u(t)\|_{X}^{2} e^{-2 \gamma t} d t\right)^{1 / 2}<\infty,
$$

and by $H^{1}\left(\mathbb{R}_{+}^{*}, X, Y, \gamma\right)$ the space of all functions $u \in$ $L_{2}\left(\mathbb{R}_{+}^{*}, X, \gamma\right)$ such that $u_{t} \in L_{2}\left(\mathbb{R}_{+}^{*}, Y, \gamma\right)$ with the norm

$$
\|u\|_{H^{1}\left(\mathbb{R}_{+}^{*}, X, Y, \gamma\right)}=\left(\|u\|_{L_{2}\left(\mathbb{R}_{+}^{*}, X, \gamma\right)}^{2}+\left\|u_{t}\right\|_{L_{2}\left(\mathbb{R}_{+}^{*}, Y, \gamma\right)}^{2}\right)^{1 / 2} .
$$

For shortness, we set

$$
\begin{gathered}
H_{B}^{m, 1}\left(Q_{T}\right)=H^{1}\left((0, T), H_{B}^{m}(G), L_{2}(G)\right), \\
L_{2}(Q, \gamma)=L_{2}\left(\mathbb{R}_{+}^{*}, L_{2}(G), \gamma\right), \\
H^{l, 0}(Q, \gamma)=L_{2}\left(\mathbb{R}_{+}^{*}, H^{l}(G), \gamma\right), \\
H^{l, 1}(Q, \gamma)=H^{1}\left(\mathbb{R}_{+}^{*}, H^{l}(G), L_{2}(G), \gamma\right), \\
V_{2, \beta}^{l, 0}(Q, \gamma)=L_{2}\left(\mathbb{R}_{+}^{*}, V_{2, \beta}^{l}(G), \gamma\right), \\
H_{\beta}^{l, 0}(Q, \gamma)=L_{2}\left(\mathbb{R}_{+}^{*}, H_{\beta}^{l}(G), \gamma\right), \\
H_{\beta}^{l, 1}(Q, \gamma)=H^{1}\left(\mathbb{R}_{+}^{*}, H_{\beta}^{l}(G), L_{2}(G), \gamma\right) .
\end{gathered}
$$

Now the definition of generalized solutions of the problem (8)-(9) is given as follows.

Definition 1. Let $f$ be a given function defined on $Q$ which belongs to $L_{2}\left(Q_{T}\right)$ for each $T>0$. A function $u \in H_{B}^{m, 1}(Q, \gamma)$ for some $\gamma \in \mathbb{R}$ is called a generalized solution of the problem (8)-(9) if and only if $\left.u\right|_{t=0}=0$, and for each $T>0$ the equality

$$
\begin{gathered}
-\int_{0}^{T} \int_{G} u_{t} \bar{\eta}_{t} d t+\sum_{|\alpha|,|\beta|=0}^{m} \int_{0}^{T} \int_{G} a_{\alpha \beta}(x, t) \partial_{x}^{\beta} u \overline{\partial_{x}^{\alpha} \eta} d x d t \\
=\int_{0}^{T} \int_{G} f \bar{\eta} d t,
\end{gathered}
$$

holds for all $\eta \in H_{B}^{m, 1}\left(Q_{T}\right)$ satisfying $\eta(\cdot, T)=0$.

\section{Statements of the Main Results}

The unique solvability of the problem is given by the following theorem.

Theorem 2. There exists a positive real number $\gamma_{0}$ such that for each $\gamma>\gamma_{0}$, if $f \in L_{2}(Q, \sigma)$ for some real number $\sigma$, the problem (8)-(9) has a unique generalized solution $u$ in the space $H_{B}^{m, 1}(Q, \gamma+\sigma)$ and

$$
\|u\|_{H_{B}^{m, 1}(\mathrm{Q}, \gamma+\sigma)}^{2} \leq C\|f\|_{L_{2}(\mathrm{Q}, \sigma)}^{2}
$$

where $C$ is a constant independent of $u$ and $f$.

The following theorem states on the regularity of the generalized solution in weighted Sobolev spaces.
Theorem 3. Let $h$ be a positive integer and $\gamma_{0}$ be the number as in Theorem 2. Suppose that the function $f$ satisfies the following conditions for some real number $\sigma$ :

$$
\begin{aligned}
& \text { (i) } f_{t^{k}} \in L_{2}\left(Q, k \gamma_{0}+\sigma\right), k \leqslant h, \\
& \text { (ii) } f_{t^{k}}(x, 0)=0,0 \leq k \leq h-1 .
\end{aligned}
$$

Let $u \in H_{B}^{m, 1}(Q, \gamma+\sigma)$ for some $\gamma>\gamma_{0}$ be the generalized solution of the problem (8)-(9). Then $u_{t^{k}} \in H_{m}^{2 m, 1}(Q,(k+2) \gamma+$ $\sigma)$ for $k=0,1, \ldots, h-1$ and

$$
\sum_{k=0}^{h-1}\left\|u_{t^{k}}\right\|_{H_{m}^{2 m, 1}(\mathrm{Q},(k+2) \gamma+\sigma)}^{2} \leqslant C \sum_{k=0}^{h}\left\|f_{t^{k}}\right\|_{L_{2}\left(\mathrm{Q}, k \gamma_{0}+\sigma\right)}^{2},
$$

where $C$ is a constant independent of $u$ and $f$.

The proofs of these theorems will be given in Section 4 . The number $\gamma_{0}$ will be defined by formula (66). It is natural that this number should be chosen as small as possible.

The remainder of this section is devoted to construct the theorem on the asymptotic behaviour of the solution near the conical point.

Let $r=|x|$ and $\omega$ be an arbitrary local coordinate system on $S^{n-1}$. Let $\delta$ be a positive real constant. A differential operator

$$
P\left(x, t, \partial_{x}\right)=\sum_{|\alpha| \leqslant l} p_{\alpha}(x, t) \partial_{x}^{\alpha}
$$

is called $\delta$-admissible operator of order $l$ near the conical point 0 if the coefficients $p_{\alpha}(x, t)$ are infinitely differentiable in $\bar{Q}$ and there is representation in a neighborhood $U$ of the conical point 0 :

$$
p_{\alpha}(x, t)=r^{|\alpha|-l} p_{\alpha}^{(0)}(\omega, t)+r^{|\alpha|-l+\delta} p_{\alpha}^{(1)}(\omega, r, t),
$$

where $p_{\alpha}^{(0)} \in C^{\infty}\left(\bar{\Omega} \times \mathbb{R}_{+}\right)$and the functions $p_{\alpha}^{(1)}$ are infinitely differentiable functions in $\bar{\Omega} \times \mathbb{R}_{+} \times \mathbb{R}_{+}$such that

$$
\left|\left(r \partial_{r}\right)^{j} \partial_{\omega}^{\beta} \partial_{t}^{k} p_{\alpha}^{(1)}(\omega, r, t)\right|<c_{j k \beta},
$$

for every multiindex $\beta$ and every pair of nonnegative integers $j, k$. Here the constants $c_{j k \beta}$ do not depend on $\omega, r$ and $t$. The leading part $\mathscr{P}$ of the operator $P$ at the point 0 is defined by

$$
\mathscr{P}\left(x, t, \partial_{x}\right)=\sum_{|\alpha| \leqslant l} r^{|\alpha|-l} p_{\alpha}^{(0)}(\omega, t) \partial_{x}^{\alpha} .
$$

It can be directly verified that the derivative $\partial_{x}^{\alpha}$ has the form

$$
\partial_{x}^{\alpha}=r^{-|\alpha|} \sum_{p=0}^{|\alpha|} p_{\alpha j}\left(\omega, \partial_{\omega}\right)(r \partial r)^{j}
$$

where $p_{\alpha j}\left(\omega, \partial_{\omega}\right)$ are differential operators of order $\leqslant|\alpha|-j$ with smooth coefficients on $\bar{\Omega}$. Thus the operator $\mathscr{P}\left(x, t, \partial_{x}\right)$ can be represented as

$$
\mathscr{P}\left(x, t, \partial_{x}\right)=r^{-l} \sum_{j=0}^{l} p_{j}\left(\omega, t, \partial_{\omega}\right)\left(r \partial_{r}\right)^{j} .
$$


For convenience we rewrite the operator $L\left(x, t, \partial_{x}\right)$ in the form

$$
L=L\left(x, t, \partial_{x}\right)=\sum_{|\alpha| \leqslant 2 m} a_{\alpha}(x, t) \partial_{x}^{\alpha}
$$

Let $\mathscr{L}=\mathscr{L}\left(x, t, \partial_{x}\right), \mathscr{B}_{j}=\mathscr{B}_{j}\left(x, t, \partial_{x}\right)(j=1, \ldots, m)$ be the leading parts of $L\left(x, t, \partial_{x}\right), B_{j}\left(x, t, \partial_{x}\right)$ at the point $x=0$. Since the coefficients of the operators $L\left(x, t, \partial_{x}\right)$ and $B_{j}\left(x, t, \partial_{x}\right)$ are smooth, it is verified easily that

$$
\begin{aligned}
\mathscr{L}\left(x, t, \partial_{x}\right) & =\sum_{|\alpha|=2 m} a_{\alpha}(0, t) \partial_{x}^{\alpha}, \mathscr{B}_{j}\left(x, t, \partial_{x}\right) \\
& =\sum_{|\alpha|=\mu_{j}} b_{j, \alpha}(0, t) \partial_{x}^{\alpha},
\end{aligned}
$$

and the operators $L\left(x, t, \partial_{x}\right)$ and $B_{j}\left(x, t, \partial_{x}\right)$ are 1 -admissible. Rewrite $\mathscr{L}\left(x, t, \partial_{x}\right), \mathscr{B}_{j}\left(x, t, \partial_{x}\right)$ in the form

$$
\begin{aligned}
\mathscr{L}\left(x, t, \partial_{x}\right) & =r^{-2 m} \mathscr{L}\left(\omega, t, \partial_{\omega}, r \partial_{r}\right), \mathscr{B}_{j}\left(x, t, \partial_{x}\right) \\
& =r^{-\mu_{j}} \mathscr{B}_{j}\left(\omega, t, \partial_{\omega}, r \partial_{r}\right) .
\end{aligned}
$$

We introduce the operator

$$
\begin{aligned}
\mathscr{U} & (\lambda, t) \\
& =\left(\mathscr{L}\left(\omega, t, \partial_{\omega}, \lambda\right), \mathscr{B}_{1}\left(\omega, t, \partial_{\omega}, \lambda\right), \ldots, \mathscr{B}_{m}\left(\omega, t, \partial_{\omega}, \lambda\right)\right),
\end{aligned}
$$

$\left(\lambda \in \mathbb{C}, t \in \mathbb{R}_{+}\right)$of the parameter-depending elliptic boundary value problem

$$
\begin{gathered}
\mathscr{L}\left(\omega, t, \partial_{\omega}, \lambda\right) u=f \text { in } \Omega \\
\mathscr{B}_{j}\left(\omega, t, \partial_{\omega}, \lambda\right) u=g_{j} \text { on } \partial \Omega, \quad j=1, \ldots, m .
\end{gathered}
$$

This is a pencil of continuous operators from

$$
\begin{aligned}
\mathscr{X} & \equiv W_{2}^{l}(\Omega) \text { into } y \\
& \equiv W_{2}^{l-2 m}(\Omega) \times \prod_{j=1}^{m} W_{2}^{l-\mu_{j}-(1 / 2)}(\partial \Omega) \quad(l \geqslant 2 m),
\end{aligned}
$$

depending polynomially on $\lambda \in \mathbb{C}$.

We mention now some well-known definitions [3]. Let $t_{0} \in \mathbb{R}_{+}$fixed. If $\lambda_{0} \in \mathbb{C}, \varphi_{0} \in \mathscr{X}$ such that $\varphi_{0} \neq 0, \boldsymbol{U}\left(\lambda_{0}, t_{0}\right) \varphi_{0}=0$, then $\lambda_{0}$ is called an eigenvalue of $\mathcal{U}\left(\lambda, t_{0}\right)$ and $\varphi_{0} \in \mathscr{X}$ is called an eigenvector corresponding to $\lambda_{0} \cdot \Lambda=$ dim ker $\boldsymbol{U}\left(\lambda_{0}, t_{0}\right)$ is called the geometric multiplicity of the eigenvalue $\lambda_{0}$.

If the elements $\varphi_{1}, \ldots, \varphi_{s}$ of $\mathscr{X}$ satisfy the equations

$$
\left.\sum_{q=0}^{\sigma} \frac{1}{q !} \frac{\partial^{\beta}}{d \lambda^{q}} \mathcal{U}\left(\lambda, t_{0}\right)\right|_{\lambda=\lambda_{0}} \varphi_{\sigma-q}=0 \text { for } \sigma=1, \ldots, s,
$$

then the ordered collection $\varphi_{0}, \varphi_{1}, \ldots, \varphi_{s}$ is said to be a Jordan chain corresponding to the eigenvalue $\lambda_{0}$ of the length $s+$ 1. The rank of the eigenvector $\varphi_{0}\left(\operatorname{rank} \varphi_{0}\right)$ is the maximal length of the Jordan chains corresponding to the eigenvector $\varphi_{0}$.

A canonical system of eigenvectors of $\boldsymbol{U}\left(\lambda_{0}, t_{0}\right)$ corresponding to the eigenvalue $\lambda_{0}$ is a system of eigenvectors $\varphi_{1,0}, \ldots, \varphi_{\Lambda, 0}$ such that rank $\varphi_{1,0}$ is maximal among the rank of all eigenvectors corresponding to $\lambda_{0}$ and $\operatorname{rank} \varphi_{j, 0}$ is maximal among the rank of all eigenvectors in any direct complement in ker $\boldsymbol{U}\left(\lambda_{0}, t_{0}\right)$ to the linear span of the vectors $\varphi_{1,0}, \ldots, \varphi_{j-1,0}(j=2, \ldots, \Lambda)$. The numbers $\kappa_{j}=$ $\operatorname{rank} \varphi_{j, 0}(j=1, \ldots, \Lambda)$ are called the partial multiplicities and the sum $\kappa=\kappa_{1}+\cdots+\kappa_{\Lambda}$ is called the algebraic multiplicity of the eigenvalue $\lambda_{0}$.

The eigenvalue of $\lambda_{0}$ is called simple if its algebraic multiplicity is equal to one.

For each fixed $t \in \mathbb{R}_{+}$the set of all complex number $\lambda$ such that $\boldsymbol{U}(\lambda, t)$ is not invertible is called the spectrum of $\mathcal{U}(\lambda, t)$. It is known that the spectrum of $\mathcal{U}(\lambda, t)$ is an enumerable set of its eigenvalues (see [3, Theorem 5.2.1]). Moreover, there are constants $\delta, R$ such that $\mathcal{U}(\lambda, t)$ is invertible for all $t \in \mathbb{R}_{+}$ and all $\lambda$ in the set

$$
\mathscr{D}:=\{\lambda \in \mathbb{C}:|\operatorname{Re} \lambda| \leqslant \delta|\operatorname{Im} \lambda|,|\lambda| \geqslant R\}
$$

(see [3, Theorem 3.6.1]).

To receive asymptotic formulas of the solutions with the coefficients regular with respect to the variable $t$ we require later that eigenvalues and eigenvectors of the pencil $\mathcal{U}(\lambda, t)$ satisfy the following assumption.

Let $l_{1}, l_{2}$ be nonnegative integers, and let $\beta_{1}, \beta_{2}$ be real numbers such that $l_{1}-\beta_{1}<l_{2}-\beta_{2}$. We say that the assumption $(\mathbf{H})$ for numbers $l_{1}, l_{2}, \beta_{1}, \beta_{2}$ is fulfilled if the following conditions are satisfied.

(i) The lines $\operatorname{Re} \lambda=-\beta_{i}+l_{i}-n / 2(i=1,2)$ do not contain eigenvalues of the pencil $\mathcal{U}(\lambda, t)$, and the strip $-\beta_{1}+l_{1}-n / 2<\operatorname{Re} \lambda<-\beta_{2}+l_{2}-n / 2$ contains the eigenvalues $\lambda_{\mu}(t), \mu=1, \ldots, N$, with the geometric multiplicities $\Lambda_{\mu}$ and the partial multiplicities $\kappa_{\mu, k}$, $\mu=1, \ldots, N, k=1, \ldots, \Lambda_{\mu}$, not depending on $t \in \mathbb{R}_{+}$. These eigenvalues are smooth functions on $\mathbb{R}_{+}$.

(ii) A canonical system

$$
\varphi_{k, s}^{(\mu)}(\omega, t), \quad k=1, \ldots, \Lambda_{\mu}, s=1, \ldots, \kappa_{\mu, k}
$$

of Jordan chains of $\boldsymbol{U}(\lambda, t)$ corresponding to the eigenvalue $\lambda_{\mu}(t)(\mu \in\{1, \ldots, N\})$ can be chosen, which consists of functions that are smooth for $t \in \mathbb{R}_{+}$ for all $\omega \in \bar{\Omega}$.

Theorem 4. Let $h$ be a positive integer. Let $\epsilon, \beta$ be real numbers such that $0 \leqslant \epsilon<1 / 2,0<\beta \leqslant m$ and $\epsilon+n / 2, \beta+n / 2$ are not integers in the case $m \geqslant n / 2$. Suppose that the assumptions of Theorem 3 and the assumption $(\mathbf{H})$ for $l_{1}=l_{2}=2 \mathrm{~m}, \beta_{1}=$ $m+\epsilon, \beta_{2}=\beta$ are fulfilled. Let $u \in H^{m, 1}(Q, \gamma)\left(\gamma>\gamma_{0}\right)$ be the generalized solution of the problem (8)-(9). Suppose further 
that the operators $L\left(x, t, \partial_{x}\right)$ and $B_{j}\left(x, t, \partial_{x}\right)$ are $(m+\epsilon-\beta)$ admissible near the origin 0 . Then,

(i) for the case $m<n / 2$, the solution $u$ admits the decomposition

$$
\begin{aligned}
u(x, t)= & \sum_{\mu=1}^{N} \sum_{k=1}^{\Lambda_{\mu}} \sum_{s=0}^{\kappa_{\mu, k}-1} c_{\mu, k, s}(t) r^{\lambda_{\mu}(t)} \\
& \times \sum_{\sigma=0}^{s} \frac{1}{\sigma !}(\ln r)^{\sigma} \varphi_{k, s}^{(\mu)}(\omega, t)+w(x, t),
\end{aligned}
$$

where $w, c_{\mu, k, s}$ are functions satisfying $w_{t^{p}} \in$ $V_{2, \beta}^{2 m, 0}(Q,(p+2) \gamma+\sigma),\left(c_{\mu, k, s}\right)_{t^{p}} \in L_{2}\left(\mathbb{R}_{+}^{*},(p+2) \gamma+\sigma\right)$ for $p=0, \ldots, h$,

(ii) for the case $m \geqslant n / 2$, we assume further that if $\lambda_{k}(t)=$ $q$ is integer for some $t \in \mathbb{R}_{+}$and some $k, 1 \leqslant k \leqslant N$, then $\lambda_{k}(t)=$ qfor all $t \in \mathbb{R}_{+}$; then the solution $u$ admits the decomposition

$u(x, t)$

$$
\begin{aligned}
& =\sum_{|\alpha| \leqslant 2 m-\ell-1} c_{\alpha}(t) x^{\alpha}+\sum_{k=2 m-\ell}^{2 m-\ell_{1}-1} r^{k} \sum_{s=0}^{\kappa_{k}} \frac{1}{s !}(\ln r)^{s} \varphi_{k, s}(\omega, t) \\
& \quad+\sum_{\mu=1}^{N} \sum_{k=1}^{\Lambda_{\mu}} \sum_{s=0}^{\kappa_{\mu, k}-1} c_{\mu, k, s}(t) r^{\lambda_{\mu}(t)} \\
& \quad \times \sum_{\sigma=0}^{s} \frac{1}{\sigma !}(\ln r)^{\sigma} \varphi_{k, s}^{(\mu)}(\omega, t)+w(x, t),
\end{aligned}
$$

where $w, c_{\alpha}, c_{\mu, k, s}$ are functions satisfying $w_{t^{p}} \in$ $V_{2, \beta}^{2 m, 0}(Q,(p+2) \gamma+\sigma),\left(c_{\alpha}\right)_{t^{p}},\left(c_{\mu, k, s}\right)_{t^{p}} \in L_{2}\left(\mathbb{R}_{+}^{*},(p+\right.$ 2) $\gamma+\sigma)$, for $p=0, \ldots, h$, and $\ell_{1}$ is the integer with $\ell_{1}-n / 2<\beta<\ell_{1}+1-n / 2, \kappa_{k}=0$ if $k$ is not an eigenvalue of $\boldsymbol{U}(\lambda, t)$; otherwise $\kappa_{k}$ is the maximal partial multiplicity of the eigenvalue $k$.

\section{Proofs of Theorems 2 and 3}

First, let us introduce some more notations. For functions $u, v$ defined in $G$ and $k \in \mathbb{N}$, we set

$$
|u|_{k, G}=\left(\int_{G} \sum_{|\alpha|=k}\left|\partial^{\alpha} u\right|^{2} d x\right)^{1 / 2}, \quad(u, v)_{G}=\int_{G} u \bar{v} d x .
$$

For functions $u$ and $v$ defined in $Q$, we set

$$
\begin{aligned}
|u|_{k, Q_{\tau}} & =\left(\int_{0}^{\tau}|u(t)|_{k, G}^{2} d t\right)^{1 / 2} \\
B_{t}(t, u, v) & =\sum_{|\alpha|,|\beta| \leqslant m} \int_{G} \frac{\partial a_{\alpha \beta}}{\partial t}(t) \partial^{\beta} u(t) \overline{\partial^{\alpha} v(t)} d x, \\
B_{t}^{\tau}(u, v) & =\int_{0}^{\tau} B_{t}(t, u, v) d t .
\end{aligned}
$$

Here and hereafter, we use $u(t)$ instead of $u(\cdot, t)$ for the shortness.

To prove Theorem 2, it is needed to introduce the Gronwall-Bellman and interpolation inequalities stated in the following lemmas.

Lemma 5. (see[14, Lemma 3.1])

Assume $u, \alpha, \beta$ are real-valued continuous on an interval $[a, b], \beta$ is nonnegative and integrable on $[a, b]$ and $\alpha$ is nondecreasing satisfying

$$
u(\tau) \leqslant \alpha(\tau)+\int_{a}^{\tau} \beta(t) u(t) d t \quad \forall a \leqslant \tau \leqslant b .
$$

Then

$$
u(\tau) \leqslant \alpha(\tau) \exp \left(\int_{a}^{\tau} \beta(t) d t\right) \quad \forall a \leqslant \tau \leqslant b
$$

Lemma 6. (see[15, Lemma 4.14])

For each positive real number $\epsilon$ and each integer $j, 0<j<$ $m$, there exists a positive real number $C=C(G, m, \epsilon)$ which is dependent only on $G, m$, and $\epsilon$ such that the inequality

$$
|u|_{j, G}^{2} \leqslant \epsilon|u|_{m, G}^{2}+C|u|_{0, G}^{2}
$$

holds for all $u \in H^{m}(G)$.

Proof of Theorem 2. The theorem is proved by repeating almost word for word the proof of Theorem 3.3 of [7]. Here we present the proof of the existence to show that the restriction of negativeness of $\sigma$ in that theorem can be omitted.

Let $\left\{\varphi_{k}\right\}_{k=1}^{\infty}$ be a basis of $H_{B}^{m}(G)$ which is orthonormal in $L_{2}(G)$. Put

$$
u^{N}(x, t)=\sum_{k=1}^{N} c_{k}^{N}(t) \varphi_{k}(x)
$$

where $\left(c_{k}^{N}(t)\right)_{k=1}^{N}$ are the solution of the system of the following ordinary differential equations of second order:

$$
\left(u_{t t}^{N}(t), \varphi_{l}\right)_{G}+B\left(t, u^{N}, \varphi_{l}\right)=\left(f(t), \varphi_{l}\right)_{G}, \quad l=1, \ldots, N,
$$

with the initial conditions

$$
c_{k}^{N}(0)=\frac{d c_{k}^{N}}{d t}(0)=0, \quad k=1, \ldots, N .
$$

Let us multiply (47) by $(d / d t) c_{k}^{N}(t)$. Take the sum with respect to $l$ from 1 to $N$ to receive

$$
\left(u_{t t}^{N}(t), u_{t}^{N}(t)\right)_{G}+B\left(\tau, u^{N}, u_{t}^{N}\right)=\left(f(t), u_{t}^{N}(t)\right)_{G} .
$$

Now adding this equality to its complex conjugate with noting that

$$
\overline{B(\tau, u, v)}=B(\tau, v, u)
$$


by the formally self-adjointness of the operator $L$, then integrating the obtained equality with respect to $t$ from 0 to $\tau$ with using the integration by parts and (48), we arrive at

$$
\left|u_{t}^{N}(\tau)\right|_{0, G}^{2}+B\left(\tau, u^{N}, u^{N}\right)=B_{t}^{\tau}\left(u^{N}, u^{N}\right)+2 \operatorname{Re}\left(f, u_{t}^{N}\right)_{Q_{\tau}} .
$$

Noting that

$$
\left|u^{N}(\tau)\right|_{0, G}^{2}=2 \operatorname{Re}\left(u^{N}, u_{t}^{N}\right)_{\mathrm{Q}_{\tau}}
$$

we have from (51) that

$$
\begin{aligned}
\left|u_{t}^{N}(\tau)\right|_{0, G}^{2}+B_{0}\left(\tau, u^{N}, u^{N}\right)+\rho|u(\tau)|_{0, G}^{2} & \\
= & B_{t}^{\tau}\left(u^{N}, u^{N}\right)+2 \rho \operatorname{Re}\left(u^{N}, u_{t}^{N}\right)_{Q_{\tau}} \\
& -\sum_{\substack{|\alpha|,|\beta| \leqslant m \\
|\alpha|+|\beta|<2 m-1}} \int_{G} a_{\alpha \beta}(\tau) \partial^{\beta} u^{N}(\tau) \overline{\partial^{\alpha} u^{N}}(\tau) d x \\
& +2 \operatorname{Re}\left(f, u_{t}^{N}\right)_{\mathrm{Q}_{\tau}} .
\end{aligned}
$$

Now we give estimations for the terms of (53). Firstly, by (2), we see that the left-hand side of (53) is greater than

$$
\left|u_{t}^{N}(\tau)\right|_{0, G}^{2}+\mu_{0}\left|u^{N}(\tau)\right|_{m, G}^{2}+\rho|u(\tau)|_{0, G}^{2}
$$

We write $B_{t}^{\tau}$ as the sum of the two following terms:

$$
\begin{aligned}
& I_{1}=\sum_{|\alpha|=|\beta|=m} \int_{Q_{\tau}} \frac{\partial a_{\alpha \beta}}{\partial t} \partial^{\beta} u^{N} \overline{\partial^{\alpha} u^{N}} d x d t, \\
& I_{2}=\sum_{\substack{|\alpha|,|\beta| \leqslant m \\
|\alpha|+|\beta| \leqslant 2 m-1}} \int_{Q_{\tau}} \frac{\partial a_{\alpha \beta}}{\partial t} \partial^{\beta} u^{N} \overline{\partial^{\alpha} u^{N}} d x d t .
\end{aligned}
$$

Put

$$
\begin{aligned}
& \mu_{1}=\sup \left\{\left|\frac{\partial a_{\alpha \beta}}{\partial t}(x, t)\right|:|\alpha|=|\beta|=m,(x, t) \in \bar{Q}\right\}, \\
& m^{\prime}=\sum_{|\alpha|=m} 1 .
\end{aligned}
$$

Then, by the Cauchy inequality, we have

$$
\begin{aligned}
\left|I_{1}\right| & \leqslant \mu_{1} \sum_{|\alpha|=|\beta|=m} \frac{1}{2}\left(\left|\partial^{\beta} u^{N}\right|_{0, Q_{\tau}}^{2}+\left|\partial^{\alpha} u^{N}\right|_{0, Q_{\tau}}^{2}\right) \\
& \leqslant m^{\prime} \mu_{1}\left|u^{N}\right|_{m, Q_{\tau}}^{2}
\end{aligned}
$$

By the Cauchy inequality and the interpolation inequality (45), for an arbitrary positive number $\epsilon_{1}$, we have

$$
\left|I_{2}\right| \leqslant \epsilon_{1}\left|u^{N}\right|_{m, Q_{\tau}}^{2}+C_{1}\left|u^{N}\right|_{0, Q_{\tau}}^{2}
$$

where $C_{1}=C_{1}\left(\epsilon_{1}\right)$ is a nonnegative constant independent of $u^{N}, f$, and $\tau$. Now using again the Cauchy and interpolation inequalities, for an arbitrary positive number $\epsilon_{2}$ with $\epsilon_{2}<\mu_{0}$, it holds that

$$
\begin{array}{|}
\left|\sum_{\substack{|\alpha|,|\beta| \leqslant m \\
|\alpha|+|\beta|<2 m-1}} \int_{G} a_{\alpha \beta} \partial^{\beta} u^{N} \overline{\partial^{\alpha} u^{N}} d x\right|^{\leqslant \epsilon_{2}\left|u^{N}(\tau)\right|_{m, G}^{2}+C_{2}\left|u^{N}(\tau)\right|_{0, G}^{2}}
\end{array}
$$

where $C_{2}=C_{2}\left(\epsilon_{2}\right)$ is a nonnegative constant independent of $u^{N}, f$, and $\tau$. Also by Cauchy inequality we have

$$
\begin{aligned}
\left|2 \rho \operatorname{Re}\left(u^{N}, u_{t}^{N}\right)_{\mathrm{Q}_{\tau}}\right| \leqslant & \frac{\left(\mu_{0}-\epsilon_{2}\right) \rho^{2}}{m^{\prime} \mu_{1}+\epsilon_{1}}\left|u^{N}\right|_{0, \mathrm{Q}_{\tau}}^{2} \\
& +\frac{m^{\prime} \mu_{1}+\epsilon_{1}}{\mu_{0}-\epsilon_{2}}\left|u_{t}^{N}\right|_{0, \mathrm{Q}_{\tau}}^{2} .
\end{aligned}
$$

Now, to deal with the last term of (53), let us consider first the case $\sigma \geqslant 0$. In this case, we use the following inequality:

$$
\left|2 \operatorname{Re}\left(f, u_{t}^{N}\right)_{\mathrm{Q}_{\tau}}\right| \leqslant \epsilon_{3}\left|u_{t}^{N}\right|_{0, \mathrm{Q}_{\tau}}^{2}+\frac{1}{\epsilon_{3}}|f|_{0, \mathrm{Q}_{\tau}}^{2}
$$

where $\epsilon_{3}>0$ arbitrary. Combining the above estimations we get from (53) that

$$
\begin{gathered}
\left|u_{t}^{N}(\tau)\right|_{0, G}^{2}+\left(\mu_{0}-\epsilon_{2}\right)\left|u^{N}(\tau)\right|_{m, G}^{2}+\left(\rho-C_{2}\right)\left|u^{N}(\tau)\right|_{0, G}^{2} \\
\leqslant\left(m^{\prime} \mu_{1}+\epsilon_{1}\right)\left|u^{N}\right|_{m, Q_{\tau}}^{2}+\left(C_{1}+\frac{\left(\mu_{0}-\epsilon_{2}\right) \rho^{2}}{m^{\prime} \mu_{1}+\epsilon_{1}}\right)\left|u^{N}\right|_{0, Q_{\tau}}^{2} \\
+\left(\frac{m^{\prime} \mu_{1}+\epsilon_{1}}{\mu_{0}-\epsilon_{2}}+\epsilon_{3}\right)\left|u_{t}^{N}\right|_{0, Q_{\tau}}^{2}+\frac{1}{\epsilon_{3}}|f|_{0, Q_{\tau}}^{2} \cdot
\end{gathered}
$$

Now fix $\epsilon_{1}, \epsilon_{2}$ and consider the function

$$
g(\rho)=\frac{C_{1}+\left(\mu_{0}-\epsilon_{2}\right) \rho^{2} /\left(m^{\prime} \mu_{1}+\epsilon_{1}\right)}{\rho-C_{2}} \text { for } \rho>C_{2} .
$$

We see that

$$
\frac{d g}{d \rho}=\frac{A \rho^{2}-2 A C_{2} \rho-C_{1}}{\left(\rho-C_{2}\right)^{2}} \quad \text { with } A=\frac{\left(\mu_{0}-\epsilon_{2}\right)}{m^{\prime} \mu_{1}+\epsilon_{1}} .
$$

We see that the function $g$ has a unique minimum at

$$
\rho_{0}=\rho_{0}\left(\epsilon_{1}, \epsilon_{2}\right)=C_{2}+\sqrt{C_{2}^{2}+\frac{C_{1}}{A}}>C_{2}
$$

Let us denote

$$
\gamma_{0}=\frac{1}{2} \inf _{\substack{\epsilon_{1}>0 \\ 0<\epsilon_{2}<\mu_{0}}} \max \left\{\frac{m^{\prime} \mu_{1}+\epsilon_{1}}{\mu_{0}-\epsilon_{2}}, g\left(\rho_{0}\right)\right\} .
$$


Now we take real numbers $\gamma, \gamma_{1}$ arbitrarily satisfying $\gamma_{0}<\gamma_{1}<$ $\gamma$. Then there are positive real numbers $\epsilon_{1}, \epsilon_{2}\left(\epsilon_{2}<\mu_{0}\right), \rho$ $\left(\rho>C_{2}\left(\epsilon_{1}, \epsilon_{2}\right)\right)$, and $\epsilon_{3}$ such that

$$
\begin{gathered}
\frac{m^{\prime} \mu_{1}+\epsilon_{1}}{\mu_{0}-\epsilon_{2}}+\epsilon_{3}<2 \gamma_{1}, \\
\frac{C_{1}\left(\epsilon_{1}, \epsilon_{2}\right)+\left(\mu_{0}-\epsilon_{2}\right) \rho^{2} /\left(m^{\prime} \mu_{1}+\epsilon_{1}\right)}{\rho-C_{2}\left(\epsilon_{1}, \epsilon_{2}\right)}<2 \gamma_{1} .
\end{gathered}
$$

From now to the end of the present proof, we fix such constants $\epsilon_{1}, \epsilon_{2}, \epsilon_{3}$, and $\rho$ and denote by $\left|\left\|u^{N}(\tau)\right\|\right|_{G}^{2}$ the lefthand side of (62). It follows from (62) and (67) that

$$
\begin{aligned}
\left|\left\|u^{N}(\tau)\right\|\right|_{G}^{2} \leqslant 2 \gamma_{1} & \int_{0}^{\tau}|\|u(t)\||_{G}^{2} d t \\
& +\frac{1}{\epsilon_{3}} \int_{0}^{\tau}|f(t)|_{0, G}^{2} d t \quad \forall \tau \geqslant 0 .
\end{aligned}
$$

By the Gronwall-Bellman inequality (44), we deduce from (68) that

$$
\begin{aligned}
\|\| u^{N}(\tau) \|\left.\right|_{G} ^{2} & \leqslant \frac{1}{\epsilon_{3}} e^{2 \gamma_{1} \tau} \int_{0}^{\tau}|f(t)|_{0, G}^{2} d t \\
& \leqslant \frac{1}{\epsilon_{3}} e^{2\left(\gamma_{1}+\sigma\right) \tau}\|f\|_{L_{2}(Q, \sigma)}^{2},
\end{aligned}
$$

for all $\tau \geqslant 0$. Here we used the fact that, for $\sigma \geqslant 0$,

$$
\begin{aligned}
\int_{0}^{\tau}|f(\cdot, t)|_{0, G}^{2} d t & =e^{2 \sigma \tau} \int_{0}^{\tau}\left|e^{-\sigma \tau} f(\cdot, t)\right|_{0, G}^{2} d t \\
& \leqslant e^{2 \sigma \tau}\|f\|_{L_{2}(Q, \sigma)}^{2} .
\end{aligned}
$$

In the case of $\sigma<0$, instead of (61), we give the following inequality

$$
\begin{aligned}
\mid 2 & \operatorname{Re}\left(f, u_{t}^{N}\right)_{\mathrm{Q}_{\tau}} \mid \\
& =\left|2 \operatorname{Re}\left(e^{-\sigma t} f, e^{\sigma t} u_{t}^{N}\right)_{\mathrm{Q}_{\tau}}\right| \\
& \leqslant \epsilon_{3}\left|e^{\sigma t} u_{t}^{N}\right|_{0, \mathrm{Q}_{\tau}}^{2}+\frac{1}{\epsilon_{3}}\left|e^{-\sigma t} f\right|_{0, Q_{\tau}}^{2} \\
& \leqslant \epsilon_{3}\left|u_{t}^{N}\right|_{0, \mathrm{Q}_{\tau}}^{2}+\frac{1}{\epsilon_{3}}\left|e^{-\sigma t} f\right|_{0, Q_{\tau}}^{2} \quad \forall \tau \geqslant 0 .
\end{aligned}
$$

Thus, by repeating the above arguments we receive (68) with the last term replaced by the last term of (71), and, therefore, we also get (69) for every real number $\sigma$.

Now multiplying both sides of this inequality by $e^{-2(\gamma+\sigma) \tau}$, then integrating them with respect to $\tau$ from 0 to $\infty$, we arrive at

$$
\|\| u^{N}\left\|_{Q, \gamma+\sigma}^{2} \leqslant \frac{1}{\epsilon_{3}}\right\| f \|_{L_{2}(\mathrm{Q}, \sigma)}^{2},
$$

where we used the notation

$$
\|\| u^{N}\left\|\left.\right|_{Q, \gamma+\sigma} ^{2}=\int_{0}^{\infty} e^{-2(\gamma+\sigma) \tau}\left|\left\|u^{N}(\tau)\right\|\right|_{G}^{2} d \tau .\right.
$$

It is clear that $|\|\cdot\||_{Q, \gamma+\sigma}$ is a norm in $H_{B}^{m, 1}(Q, \gamma+\sigma)$ which is equivalent to the norm $\|\cdot\|_{H_{B}^{m, 1}(Q, \gamma+\sigma)}$. Thus, it follows from (72) that

$$
\left\|u^{N}\right\|_{H_{B}^{m, 1}(\mathrm{Q}, \gamma+\sigma)}^{2} \leqslant C\|f\|_{L_{2}(\mathrm{Q}, \sigma)}^{2},
$$

where $C$ is a constant independent of $f$ and $N$.

From the inequality (74), by standard weak convergence arguments (see, e.g., [16, Ch. 7]), we can conclude that the sequence $\left\{u^{N}\right\}_{N=1}^{\infty}$ possesses a subsequence convergent to a function $u \in H_{B}^{m, 1}(Q, \gamma+\sigma)$ which is a generalized solution of problem (8)-(9). Moreover, it follows from (74) that the inequality (22) holds.

Now we are going to prove Theorem 3. First, we give some needed auxiliary lemmas. The first lemma deals with the regularity of the solution with respect to time variable. It is proved by repeating almost word for word the proof of Theorem 3.4 of [7] with noting that, as in Theorem 2, the assumption $\sigma \geqslant 0$ in [7] can be removed.

Lemma 7. Suppose that all the assumptions of Theorem 3 are fulfilled. Then $u_{t^{k}} \in H_{B}^{m, 1}(Q,(k+1) \gamma+\sigma)$ for $k=0,1, \ldots, h$ and

$$
\sum_{k=0}^{h}\left\|u_{t^{k}}\right\|_{H_{B}^{m, 1}(\mathrm{Q},(k+1) \gamma+\sigma)}^{2} \leq C \sum_{k=0}^{h}\left\|f_{t^{k}}\right\|_{L_{2}\left(\mathrm{Q}, k \gamma_{0}+\sigma\right)}^{2},
$$

where $C$ is a constant independent of $u$ and $f$. lemma.

From the proof of [12, Lemma 5.3] we have the following

Lemma 8. Let $f \in H_{m}^{0}(G), g_{j} \in H_{m}^{2 m-\mu_{j}-1 / 2}(\Gamma), j=1, \ldots, m$, and $t_{0} \in \mathbb{R}_{+}^{*}$. Suppose that $u \in H_{B}^{m}(G) \cap H_{\mathrm{loc}}^{2 m}(\bar{G} \backslash\{0\})$ is a solution of the following problem:

$$
\begin{gathered}
L\left(x, t_{0}, \partial\right) u=f \text { in } G \\
B_{j}\left(x, t_{0}, \partial\right) u=g_{j} \text { on } \Gamma, \quad j=1, \ldots, m .
\end{gathered}
$$

Then $u \in H_{m}^{2 m}(G)$ and

$$
\begin{aligned}
& \|u\|_{H_{m}^{2 m}(G)}^{2} \\
& \quad \leqslant C\left(\|f\|_{H_{m}^{0}(G)}^{2}+\sum_{j=1}^{m}\left\|g_{j}\right\|_{H_{m}^{2 m-\mu_{j}-1 / 2}(\Gamma)}^{2}+\|u\|_{H_{B}^{m}(G)}^{2}\right),
\end{aligned}
$$

where the constant $C$ is independent of $u, f$, and $t_{0}$.

Proof of Theorem 3. According to Lemma 7 we have

$$
u_{t^{k}} \in H_{B}^{m, 1}(Q,(k+1) \gamma+\sigma), \quad k \leqslant h .
$$

Moreover, as in proof of Theorem 4.1 of [7], we have

$$
\begin{array}{r}
u_{t^{k}}(\cdot, t) \in H_{\mathrm{loc}}^{2 m}(\bar{G} \backslash\{0\}) \text { for a.e. } t \in \mathbb{R}_{+}^{*} \\
\text { and all } k \leqslant h-1 .
\end{array}
$$


Now we prove the theorem by induction on $h$. By (78), $u_{t t} \in L_{2}(Q, 2 \gamma+\sigma)$. Thus, from (21) we have

$$
B(t, u, \eta)=\left(f(t)-u_{t t}(t), \eta\right)
$$

for all $\eta \in H_{B}^{m}(G)$ and a.e. $t \in \mathbb{R}_{+}^{*}$. Since $f_{1}(t):=f(t)-u_{t t}(t) \in$ $L_{2}(G) \subset H_{m}^{0}(G)$ for a.e. $t \in \mathbb{R}_{+}^{*}$, by Lemma 8 , we get from $(80)$ that $u(t) \in H_{m}^{2 m}(G)$ for a.e. $t \in \mathbb{R}_{+}^{*}$ and

$$
\|u(t)\|_{H_{m}^{2 m}(G)}^{2} \leqslant C\left(\left\|f_{1}(t)\right\|_{L_{2}(G)}^{2}+\|u(t)\|_{H^{m}(G)}^{2}\right),
$$

where $C$ is a constant independent of $u, f_{1}$, and $t$. Now multiplying both sides of (81) with $e^{-2(2 \gamma+\sigma) t}$, then integrating with respect to $t$ from 0 to $\infty$ and using estimates from Lemma 7, we obtain

$$
\|u\|_{H_{m}^{2 m, 1}(\mathrm{Q}, 2 \gamma+\sigma)}^{2} \leqslant C\|f\|_{L_{2}(\mathrm{Q}, \sigma)}^{2},
$$

where $C$ is a constant independent of $u$ and $f$. Hence, the theorem is valid for $h=1$.

Assume that the theorem is true for some nonnegative $h-$ 2. We will prove it for $h-1$. Differentiating $h-1$ times both sides of (80) with respect to $t$ we have

$$
\begin{aligned}
B\left(t, u_{t^{h-1}}, \eta\right)= & \left(f_{t^{h-1}}(t), \eta\right)-\left(u_{t^{h+1}}(t), \eta\right) \\
& -\sum_{k=0}^{h-2}\left(\begin{array}{l}
h \\
k
\end{array}\right) B_{t^{h-k-1}}\left(t, u_{t^{k}}, \eta\right),
\end{aligned}
$$

for all $\eta \in H_{B}^{m}(G)$, a.e. $t \in \mathbb{R}_{+}^{*}$. From (6) it follows that

$$
B_{t^{k}}(t, v, \eta)=\left(L_{t^{k}}(x, t, \partial) v, \eta\right)
$$

for all $v \in H_{B}^{m}(G) \cap H_{m}^{2 m}(G)$ and $\eta \in H_{B}^{m}(G)$. Thus, from (83) we deduce

$$
B\left(t, u_{t^{h}}, \eta\right)=\left(F_{h}(\cdot, t), \eta\right)
$$

for all $\eta \in H_{B}^{m}(G)$ and a.e. $t \in \mathbb{R}_{+}$, where

$$
\begin{aligned}
F_{h}= & f_{t^{h}}(\cdot, t)-u_{t^{h+2}}(\cdot, t) \\
& -\sum_{k=0}^{h-1}\left(\begin{array}{l}
h \\
k
\end{array}\right) L_{t^{h-k}}(\cdot, t, D) u_{t^{k}} \in H_{m}^{0}(G) .
\end{aligned}
$$

By the induction assumption, it holds that

$$
\begin{array}{r}
u_{t^{k}} \in H_{m}^{2 m, 1}(Q,(k+2) \gamma+\sigma) \subset H_{m}^{2 m, 1}(Q,(h+1) \gamma+\sigma), \\
k=0, \ldots, h-2 .
\end{array}
$$

Moreover,

$$
f_{t^{h-1}} \in L_{2}(Q,(h-1) \gamma+\sigma) \subset L_{2}(Q,(h+1) \gamma+\sigma)
$$

by the assumption of the theorem, and

$$
u_{t^{h+1}} \in L_{2}(Q,(h+1) \gamma+\sigma)
$$

by Lemma 7. Thus, for a.e. $t \in \mathbb{R}_{+}^{*}$, we have $\tilde{f}(t) \in H_{m}^{0}(G)$, $\widetilde{g}(t) \in H_{\alpha}^{j-(1 / 2)}(\Gamma)$, and

$$
\begin{aligned}
& \|\tilde{f}(t)\|_{H_{m}^{0}(G)}^{2} \\
& \quad \leqslant C\left(\left\|f_{t^{h-1}}(t)\right\|^{2}+\left\|u_{t^{h+2}}(t)\right\|_{L_{2}(G)}^{2}+\sum_{k=0}^{h-2}\left\|u_{t^{k}}(t)\right\|_{H_{m}^{2 m}(G)}^{2}\right),
\end{aligned}
$$

where $C$ is the constant independent of $u, f$ and $t$. Now we can repeat the arguments above to conclude that $u_{t^{h-1}} \in$ $H_{m}^{2 m, 1}(Q,(h+1) \gamma+\sigma)$ with the estimate $(23)$ for $k=h-1$. The proof is completed.

\section{The Proof of Theorem 4}

Let us first give some auxiliary lemmas.

Lemma 9. Let $l$ be a nonnegative integer, $l \geqslant 2 m$, and let $\beta_{1}, \beta_{2}$ be real numbers, $\beta_{1}>\beta_{2}$. Suppose that the assumption $(\mathbf{H})$ is fulfilled for the numbers $l_{1}=l_{2}=l, \beta_{1}, \beta_{2}$. Let $h \in \mathbb{N}$, let $\gamma_{0} \leqslant$ $\gamma_{1} \leqslant \cdots \leqslant \gamma_{h}$ be real numbers, and let $f$ and $g_{j}$ be functions satisfying $f_{t^{p}} \in V_{2, \beta_{2}}^{l-2 m, 0}\left(Q, \gamma_{p}\right),\left(g_{j}\right)_{t^{p}} \in V_{2, \beta_{2}}^{l-\mu_{j}-(1 / 2), 0}\left(S, \gamma_{p}\right)$ for $p=0, \ldots, h$. Suppose that $u \in V_{2, \beta_{1}}^{l, 0}\left(Q, \gamma_{0}\right)$ is a solution of the problem

$$
\begin{gathered}
\mathscr{L}\left(t, \partial_{x}\right) u=f \text { in } Q, \\
\mathscr{B}_{j}\left(t, \partial_{x}\right) u=g_{j} \text { on } S, \quad j=1, \ldots, m .
\end{gathered}
$$

Then $u$ admits the following representation:

$$
\begin{aligned}
u(x, t)= & \sum_{\mu=1}^{N} \sum_{k=1}^{\Lambda_{\mu}} \sum_{s=0}^{\kappa_{\mu, k}-1} c_{\mu, k, s}(t) r^{\lambda_{\mu}(t)} \\
& \times \sum_{\sigma=0}^{s} \frac{1}{\sigma !}(\ln r)^{\sigma} \varphi_{k, s}^{(\mu)}(\omega, t)+w(x, t),
\end{aligned}
$$

where $w$ and $c_{\mu, k, s}$ are functions satisfying $w_{t^{p}} \in V_{2, \beta_{2}}^{l, 0}\left(Q, \gamma_{p}\right)$, $\left(c_{\mu, k, s}\right)_{t^{p}} \in L_{2}\left(\mathbb{R}_{+}^{*}, \gamma_{p}\right)$ for $p=0, \ldots, h$.

Proof. From the proof of Lemma 4.5 of [13] (see also [17, Lemma 4.1]), it is known that, for each fixed $t \in \mathbb{R}_{+}^{*}$, the solution $u(x, t)$ admits the representation (92) and the following inequality

$$
\begin{aligned}
& \max \left(\left\|w_{t^{p}}(\cdot, t)\right\|_{V_{2, \beta_{2}}^{l}(G)}^{2},\left|\left(c_{\mu, k, s}\right)_{t^{q}}(t)\right|\right) \\
& \leqslant C\left(\sum_{q=0}^{p}\left\|f_{t^{q}}(\cdot, t)\right\|_{V_{2, \beta_{2}}^{l-2 m}(G)}^{2}\right. \\
& \left.+\sum_{j=1}^{m} \sum_{q=0}^{p}\left\|\left(g_{j}\right)_{t^{q}}(\cdot, t)\right\|_{V_{2, \beta_{2}}^{l-\mu_{j}-1 / 2}(\Gamma)}^{2}\right),
\end{aligned}
$$


holds for all $t \in \mathbb{R}_{+}^{*}, p=0,1, \ldots, h$, where $C$ is a constant independent of $u, f, g_{j}$, and $t$. Now multiplying both sides of (93) by $e^{-2 \gamma_{p} t}$, then integrating with respect to $t$ from 0 to $\infty$, we see that $w_{t^{p}} \in V_{2, \beta_{2}}^{l_{2}, 0}\left(Q, \gamma_{p}\right),\left(c_{\mu, k, s}\right)_{t^{p}} \in L_{2}\left(\mathbb{R}_{+}^{*}, \gamma_{p}\right)$. The lemma is proved.

By applying Lemma 9 and repeating the arguments in the proof of [13, Lemma 4.6], we get the following lemma.

Lemma 10. Suppose that all assumptions of Lemma 9 are fulfilled. Suppose further that the operators $L\left(x, t, \partial_{x}\right)$ and $B_{j}\left(x, t, \partial_{x}\right)$ are $\delta$-admissible near the origin 0 , where $\delta=\beta_{1}-$ $\beta_{2}$. Let $u \in V_{2, \beta_{1}}^{l_{1}, 0}\left(Q, \gamma_{0}\right)$ be a solution of the problem

$$
\begin{gathered}
L\left(x, t, \partial_{x}\right) u=f \text { in } \mathrm{Q}, \\
B_{j}\left(x, t, \partial_{x}\right) u=g_{j} \text { on } S, \quad j=1, \ldots, m .
\end{gathered}
$$

Then $u$ admits a representation of the form (92).

The following lemma follows directly from the proof of [13, Lemma 4.7].

Lemma 11. Let $h \in \mathbb{N}$, and let $\gamma_{0} \leqslant \gamma_{1} \leqslant \cdots \leqslant \gamma_{h}$ be real numbers. Let

$$
\begin{gathered}
f=r^{\lambda_{0}(t)-2 m} \tilde{f}, \\
g_{j}=r^{\lambda_{0}(t)-\mu_{j}} \widetilde{g}_{j}, \quad j=1, \ldots, m,
\end{gathered}
$$

where $\tilde{f}$ and $\widetilde{g}_{j}$ are given functions defined on $\Omega \times \mathbb{R}_{+}^{*}, \partial \Omega \times$ $\mathbb{R}_{+}$, respectively, satisfying $(\tilde{f})_{t^{p}} \in L_{2}\left(\Omega \times \mathbb{R}_{+}^{*}, \gamma_{p}\right),\left(\widetilde{g}_{j}\right)_{t^{p}} \in$ $L_{2}\left(\partial \Omega \times \mathbb{R}_{+}, \gamma_{p}\right), j=1, \ldots, m, p=0, \ldots, h$. Suppose that if $\lambda_{0}(t)$ is an eigenvalue of $\mathcal{U}(\lambda, t)$ for some $t$, then it is an eigenvalue of $\mathcal{U}(\lambda, t)$ for all $t \in \mathbb{R}_{+}$with the geometric multiplicity and the partial multiplicities not depending on $t \in$ $\mathbb{R}_{+}$. Then there exists a solution $u$ of the problem (91) which has the form

$$
u=r^{\lambda_{0}(t)} \sum_{\sigma=0}^{\kappa} \frac{1}{\sigma !}(\ln r)^{\sigma} u_{\kappa-\sigma}
$$

where $u_{\sigma}$ are functions defined on $\Omega \times \mathbb{R}_{+}^{*}$ satisfying $\left(u_{\sigma}\right)_{t^{p}} \in$ $L_{2}\left(\mathbb{R}_{+}^{*}, H^{2 m}(\Omega), \gamma_{p}\right), \sigma=0, \ldots, \kappa$. Here $\kappa=0$ if $\lambda_{0}(t)$ is not an eigenvalue of $\mathcal{U}(\lambda, t)$; otherwise $\kappa$ is the maximal partial multiplicity of $\lambda_{0}(t)$.

Proof of Theorem 4. According to Theorem 3, we have

$$
u_{t^{p}} \in H_{m}^{2 m, 1}(Q,(k+2) \gamma+\sigma) \subset H_{m+\epsilon}^{2 m, 1}(Q,(k+2) \gamma+\sigma) \text {, }
$$

for $p=0, \ldots, h-1$.

Rewrite (8) in the form

$$
\begin{gathered}
L u=f_{1}:=f-u_{t t} \text { in } Q, \\
B_{j} u=0 \text { on } S, \quad j=1, \ldots, m .
\end{gathered}
$$

Since $u_{t^{p+2}} \in L_{2}(Q,(p+2) \gamma+\sigma)$ by $(75)$ and $f_{t^{p}} \in L_{2}(Q, p \gamma+\sigma)$ by the assumption, we have

$$
\begin{aligned}
& \left(f_{1}\right)_{t^{p}} \in L_{2}(Q,(p+2) \gamma+\sigma) \\
& \quad \equiv V_{2,0}^{0,0}(Q,(p+2) \gamma+\sigma) \subset V_{2, \beta}^{0,0}(Q,(p+2) \gamma+\sigma)
\end{aligned}
$$

for $p=0, \ldots, h-1$.

Now the assertions of the theorem are obtained by applying Lemma 10 and repeating almost word for word the proof of [13, Theorem 4.8].

\section{Acknowledgment}

This research is funded by the Vietnam National Foundation for Science and Technology Development (NAFOSTED) under Grant no. 101.01-2011.30.

\section{References}

[1] M. Dauge, Elliptic Boundary Value Problems on Corner Domains, vol. 1341 of Lecture Notes in Mathematics, Springer, Berlin, Germany, 1988.

[2] V. A. Kondrat'ev, "Boundary value problems for elliptic equations in domains with conical or angular points," Trudy Moskovskogo Matematičeskogo Obščestva, vol. 16, pp. 209292, 1967, English translation: Transactions of the Moscow Mathematical Society, vol. 16, 1967.

[3] V. A. Kozlov, V. G. Maz'ya, and J. Rossmann, Elliptic Boundary Value Problems in Domains with Point Singularities, vol. 52 of Mathematical Surveys and Monographs, American Mathematical Society, Providence, RI, USA, 1997.

[4] A. Y. Kokotov and B. A. Plamenevskiŭ, "On the cauchy-Dirichlet problem for a hyperbolic system in a wedge," Algebra i Analiz, vol. 11, no. 3, pp. 140-195, 1999.

[5] A. Y. Kokotov and B. A. Plamenevskiu, "On the asymptotics of solutions to the Neumann problem for hyperbolic systems in domains with conical points," Algebra i Analiz, vol. 16, no. 3, pp. 56-98, 2004, English translation: Petersburg Mathematical Journal, Vol. 16, no. 3, pp. 477-506, 2005.

[6] N. M. Hung, "Asymptotic behaviour of solutions of the first boundary-value problem for strongly hyperbolic systems near a conical point at the boundary of the domain," Matematicheskil Sbornik, vol. 190, no. 7, pp. 103-126, 1999, English translation: Sbornik: Mathematics, vol. 190, no. 7, pp. 1035-1058, 1999.

[7] N. M. Hung, N. T. Anh, and P. K. Chuc, "On the regularity of the solution for the second initial boundary value problem for hyperbolic systems in domains with conical points," Boundary Value Problems, vol. 2011, article 17, 2011.

[8] N. M. Hung and B. T. Kim, "On the solvability of the first mixed problem for strongly hyperbolic system in infinite nonsmooth cylinders," Taiwanese Journal of Mathematics, vol. 12, no. 9, pp. 2601-2618, 2008.

[9] N. M. Hung and J. C. Yao, "Cauchy-Dirichlet problem for second-order hyperbolic equations in cylinders with nonsmooth base," Nonlinear Analysis: Theory Methods \& Applications, vol. 70, no. 2, pp. 741-756, 2009.

[10] M. S. Agranovich, "Elliptic boundary problems," in Partial Differential Equations, M. S. Agranovich, Yu. V. Egorov, and 
M. A. Shubin, Eds., vol. 79 of Encyclopaedia of Mathematical Sciences, pp. 1-144, Springer, Berlin, Germany, 1997.

[11] J. Rossmann, "The asymptotics of the solutions of elliptic variational problems in domains with edges on the boundary," Zeitschrift für Analysis und ihre Anwendungen, vol. 9, no. 3, pp. 565-575, 1990.

[12] N. M. Hung and N. T. Anh, "Regularity of solutions of initialboundary value problems for parabolic equations in domains with conical points," Journal of Differential Equations, vol. 245, no. 7, pp. 1801-1818, 2008.

[13] N. M. Hung and N. T. Anh, "The initial-boundary value problems for parabolic equations in domains with conical points," in Advances in Mathematics Research, A. R. Baswell, Ed., vol. 10, chapter 4, pp. 205-247, Nova Science Publishers, NewYork, NY, USA, 2009.

[14] J. Hale, Theory of Functional Differential Equations, Springer, New York, NY, USA, 1977.

[15] R. A. Adams, Sobolev Spaces, Academic Press, 1975.

[16] L. C. Evans, Partial Differential Equations, vol. 19 of Graduate Studies in Mathematics, American Mathematical Society, Providence, RI, USA, 1998.

[17] N. M. Hung and N. T. Anh, "Asymptotic formulas for solutions of parameter-depending elliptic boundary-value problems in domains with conical points," Electronic Journal of Differential Equations, vol. 2009, no. 125, pp. 1-21, 2009. 


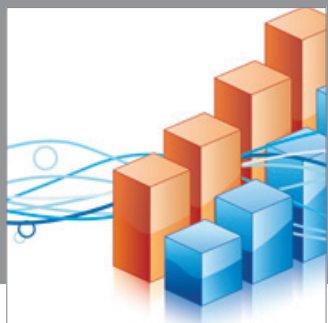

Advances in

Operations Research

mansans

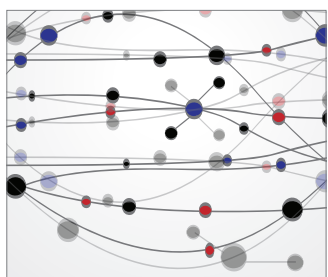

The Scientific World Journal
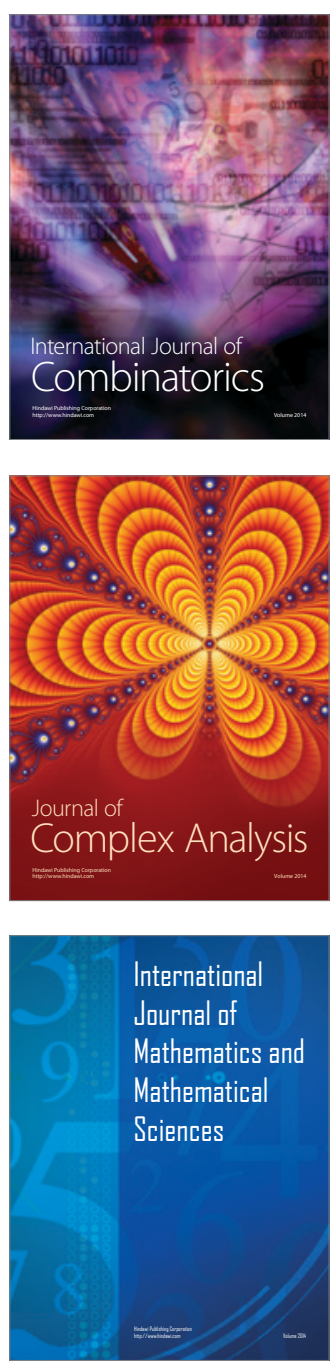
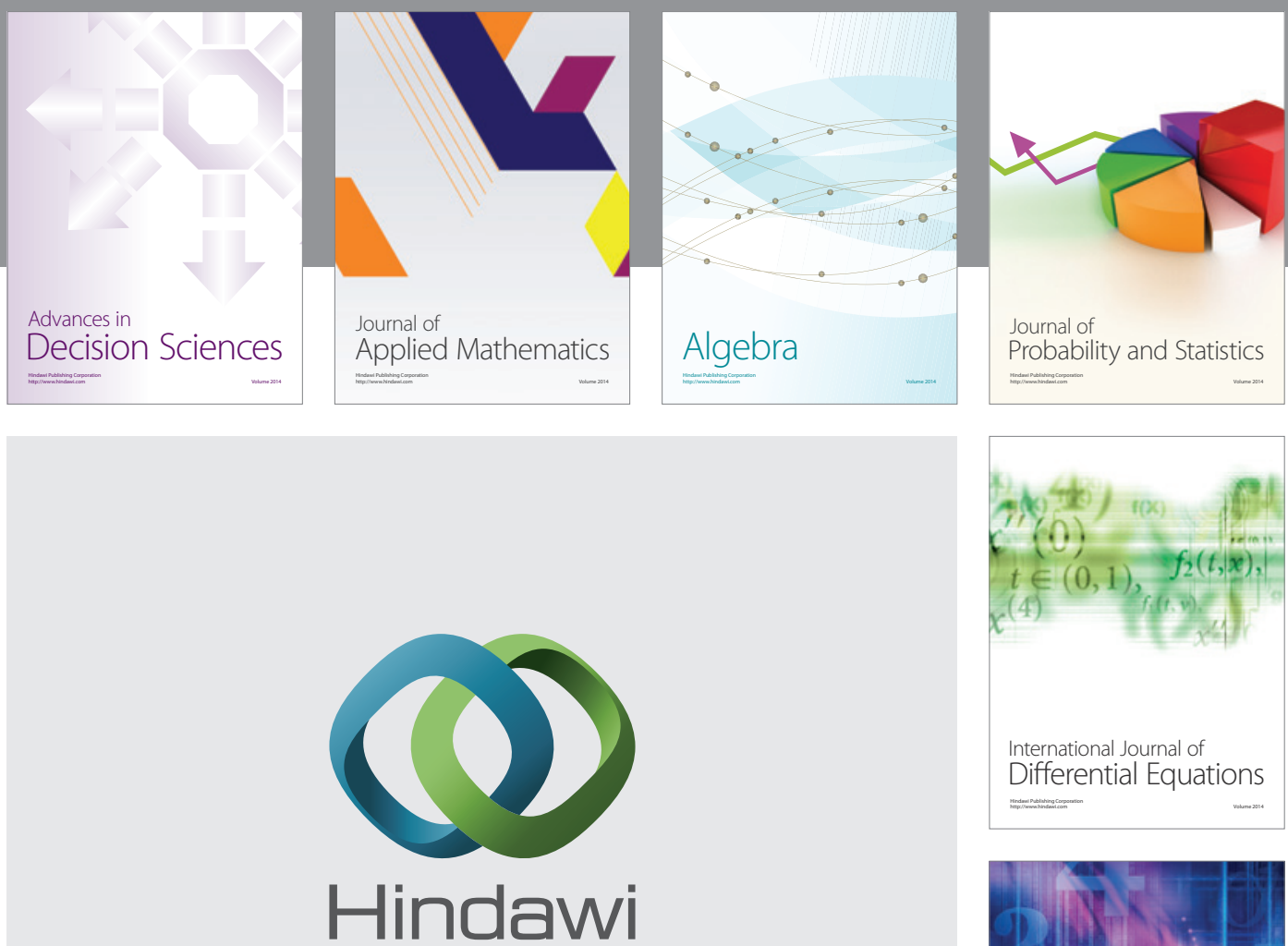

Submit your manuscripts at http://www.hindawi.com
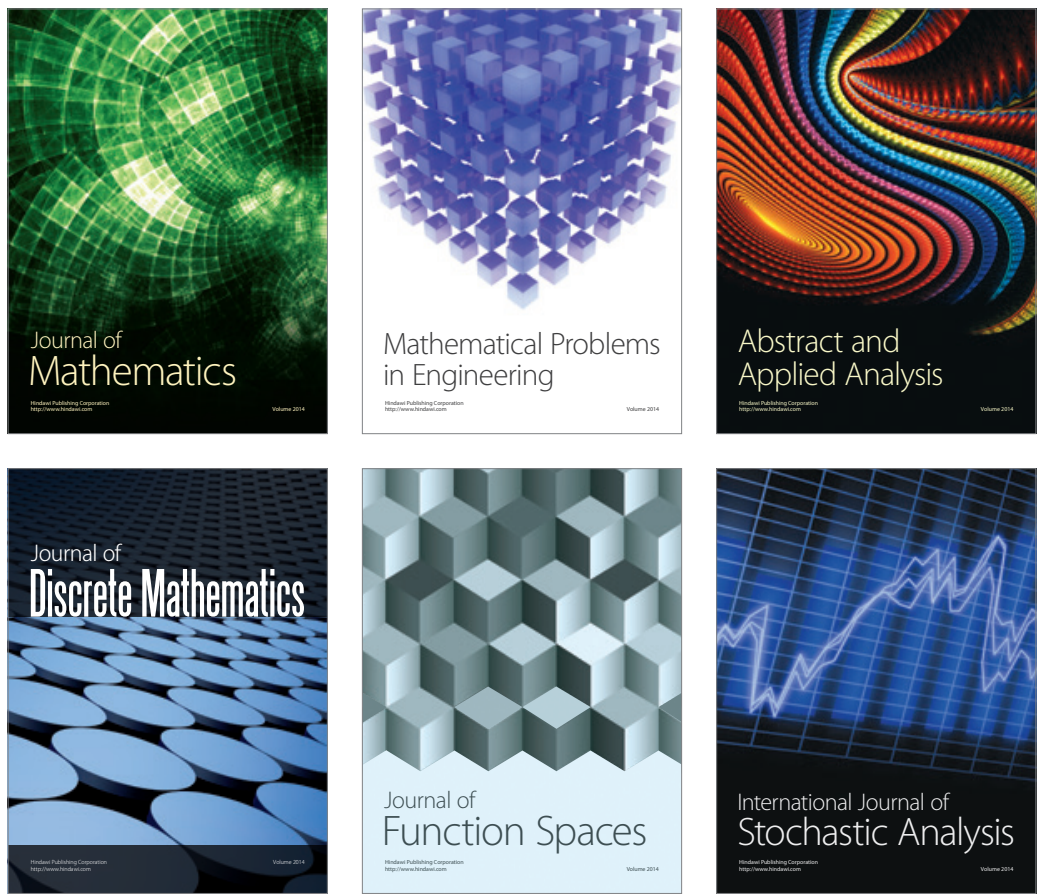

Journal of

Function Spaces

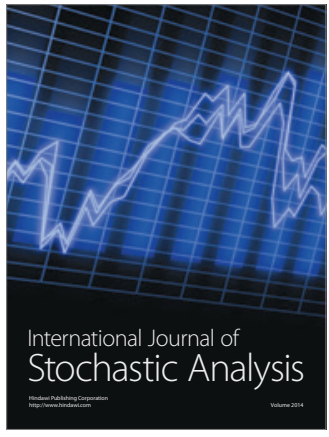

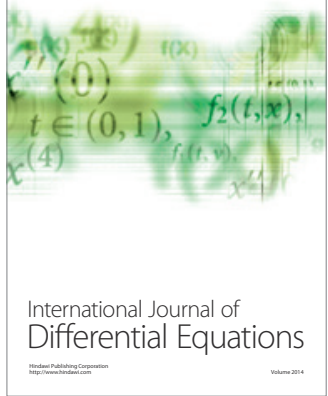
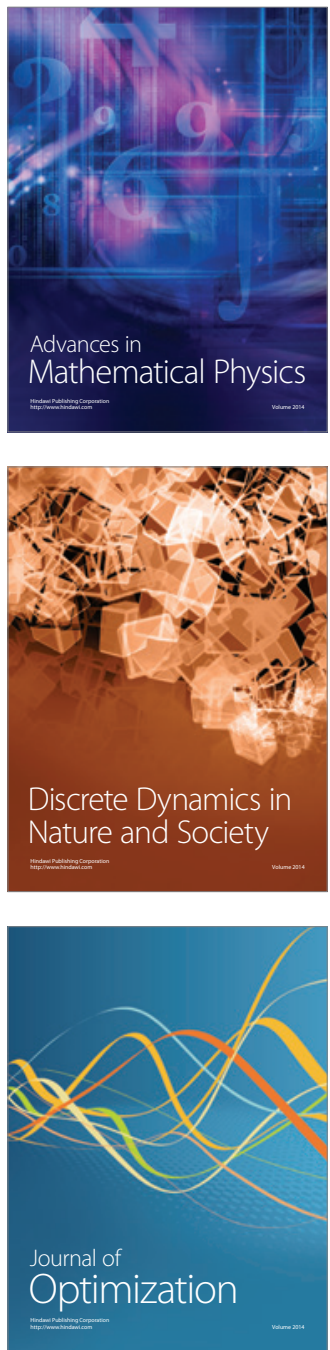